\title{
CARACTERIZACIÓN COMPARATIVA DE LA CONDICIÓN LIMNOLÓGICA DEL LAGO GUAMUÉZ EN RELACIÓN CON LA PRODUCCIÓN DE TRUCHA ARCOIRIS (ONCORHYNCHUS MYKISSI EN JAULAS FLOTANTES
}

\section{COMPARATIVE CHARACTERIZATION OF THE LIMNOLOGICAL STATUS OF LAKE GUAMEZ IN RELATION TO THE PRODUCTION OF RAINBOW TROUT (ONCORHYNCHUS MYKISSI IN FLOATING CAGES}

\author{
Jorge Nelson López Macías*, Julbrinner Salas Benavides**
}

\begin{abstract}
Resumen
La presente investigación fue realizada por el Grupo de Investigaciones en Acuacultura de la Universidad de Nariño (GIAC), con financiación de la Vicerrectoría de Investigaciones y Posgrados y la Corporación Autónoma Regional de Nariño (CORPONARIÑO). El estudio evaluó durante 90 días, los parámetros limnológicos del lago Guamuéz, con el propósito de establecer, el efecto de la producción de trucha arcoiris en jaulas flotantes, en la eutrofización de este cuerpo de agua. Para este efecto se efectuaron 14 muestreos, en ocho estaciones principales ubicadas en diferentes zonas perimetrales del lago. Se utilizo una sonda electrométrica de profundidad, para determinar temperatura, oxígeno disuelto, $\mathrm{pH}$, nitratos a diferentes alturas de la columna de agua. Así mismo, se determino, índice de turbidez y transparencia con Disco Secchi. Posteriormente, en los laboratorios especializados de la Universidad de Nariño y en el Laboratorio de Calidad de Aguas del Programa de Ingeniería Acuícola, se caracterizó cuantitativa y cualitativamente la comunidad planctónica y bentónica, se determinó según los protocolos establecidos por «Standard Methods for Examinations of Water and Wastes», Demanda Bioquímica de Oxígeno (DBO5), concentración de sólidos, nitritos, Fósforo total y amonio y se efectuaron los análisis de coliformes totales y fecales. Los resultados del ensayo demuestran que la transparencia es menor en todos los sitios de muestreo, en el centro de las jaulas. Las estaciones con mayor turbidez, corresponden a las explotaciones intensivas, o las localizadas cerca de asentamientos urbanos como es el caso de la E8 ubicada la vereda El Puerto, población que vierte directamente en el cauce del río El Encano, las aguas servidas que finalmente llegan al lago. Igualmente el mayor número de coliformes totales y fecales se registró en las granjas de trucha próximas a la desembocadura de este río. La relación Nitrógeno Total: Fósforo Total, durante el periodo de observación fue de 2,6:1, lo que señala al fósforo como elemento nutricional limitante para una futura eutrofia del lago. Sin embargo se detectan niveles altos de nitrógeno en los sitios de muestreo cerca a la desembocadura de afluentes localizados en áreas deforestadas como sucede con el río El encano y las quebradas Moras, Romerillo y Quilinza, a las cuales, les llega aguas de escorrentía que arrastran gran cantidad de materia orgánica, nutrientes y fertilizantes, procedentes de cultivos limpios de mora, papa y explotaciones pecuarias. También se observa incremento del aporte de nitrógeno y cantidad de materiales en suspensión en los sitios aledaños a la carretera perimetral del lago. El análisis de Pearson demuestra correlaciones altamente significativas de cianofitas, rotíferos y
\end{abstract}

Artículo recibido: 12/01/2013 Aprobado: 21/04/2013

* PhD en biología. Profesor Titular Universidad de Nariño. Email: jorgelopezmacias@gmail.com

** MSc en biología. Profesor Titular Universidad de Nariño. Email: biojull77@hotmail.com. 
copépodos con respecto al fósforo total $(0,74)$. Igualmente, correlación positiva entre protozoarios y clorofila - a $(0,81)$. Las categorías biológicas del bentos están correlacionadas con sólidos disueltos de fondo $(0,64)$ y turbidez $(0.73))$. Las cianofitas registran correlación positiva con nitratos $(0.76)$, sólidos totales $(0,78)$, sólidos suspendidos $(0,78)$, sólidos volátiles $(0,76)$. Los resultados de este estudio, comparados con los reportados por Castillo y Martínez (1996), demuestran que no existe un deterioro negativo, en la caracterización fisicoquímica y trófica, causado por las actividades acuícolas desarrollada en los últimos 12 años, debido a la capacidad de autopurificación del lago Guamuéz, por su condición polimíctica temporal. Sin embargo, esta capacidad estaría limitada en el futuro por la situación oligotrófica, de este cuerpo de agua. Por esta razón, los resultados de esta investigación, indican que se debe implementar de manera urgente un plan de ordenamiento integral del lago Guamuéz que incluya todas las actividades pesqueras, acuícolas, forestales, agrícolas, pecuarias y la disposición sanitaria de aguas servidas, procedentes de los distintos asentamientos urbanos, localizados en la zona de influencia para disminuir el aporte de materia orgánica en este importante lago de altiplano.

Palabras clave: Trucha arcoíris, parámetros limnológicos, trofización y Acuacultura.

\section{Abstract}

This research was conducted by the Aquaculture Research Group of the University of Nariño (GIAC), with funding from the office of the Vice Chancellor of Research and Post-graduate Studies and the Corporación Autónoma Regional de Nariño (CORPONARIÑO). The study evaluated for 90 days, the limnological parameters of the Lake Guamuez, in order to establish the effect of rainbow trout production in floating cages in the eutrophication of this body of water. For this purpose, 14 samples were conducted in eight major stations located in different areas of the lake perimeter. An electrometric depth probe was used to determine temperature, dissolved oxygen, $\mathrm{pH}$, and nitrates at different levels of the water column. Index of turbidity and transparency by means of Secchi Disk was also determined. Subsequently, in the specialized laboratories of the University of Nariño and the laboratory of quality of waters and wastes of the program of aquaculture engineering, the planktonic and benthic community was quantitatively and qualitatively characterized; It was determined according to established protocols by "Standard Methods for Examinations of Water and Wastes", the Biochemical Oxygen Demand (BOD5), the concentration of solids, nitrite, total phosphorus and ammonium, and an analyses of total and faecal coliforms were made. Test results show that transparency is lower in all sampling sites in the center of the cages. Stations with higher turbidity correspond to intensive exploitation, or those located near urban settlements such as the E8, located in the village of El Puerto which pours directly into the river El Encano, wastewater that eventually reaches the Lake. Likewise, the largest number of total and fecal coliforms was recorded in trout farms near the mouth of this river. The relationship Total Nitrogen: Total Phosphorus during the period of observation was 2.6:1, indicating phosphorus as a limiting nutrient for a future eutrophication of the lake. However, high nitrogen levels were detected at sampling sites near the mouth of tributaries located in deforested areas, as in the river The Encano, and streams Moras, Romerillo and Quilinza, which receive runoff which carry a large amount of organic matter, nutrients and fertilizers, from clean crops of blackberries and potatoes, and from livestock farms. An increase in the contribution of nitrogen and the amount of materials in suspension in sites adjacent to the road perimeter of the Lake is also observed. Pearson analysis demonstrated highly significant correlations of cyanobacteria, rotifers and copepods with respect to total phosphorus $(0.74)$. Similarly, a positive correlation between protozoa and chlorophyll - (0.81), was observed. The benthic biological categories are correlated with dissolved solids from the bottom of the lake (0.64) and turbidity (0.73)). The cyanophytes recorded positive correlation with nitrates $(0.76)$, total solids $(0.78)$, suspended solids (0.78), and volatile solids (0.76). The results of this study, compared with those reported by Castillo and Martinez (1996) show that there is no negative deterioration in the trophic and physicochemical characterization, caused by the aquaculture activities developed in the last 12 years, due to the ability of self-purification of Guamuez Lake, due to its temporary polymict condition. However, this capacity would 
be limited in the future by the oligotrophic status of this body of water. For this reason, the results of this research indicate that a comprehensive management plan of Guamuez Lake must be implemented, urgently. This plan must include all fishing, aquaculture, forestry, agricultural and livestock activities, and sanitary disposal of sewage from the various urban settlements located in the zone of influence in order to diminish the contribution of organic matter in this important highland lake.

Keywords: rainbow trout, limnological parameters, eutrophication, Aquaculture.

\section{Introducción}

El estudio de los lagos como fuentes de recursos naturales de diversa índole constituye una de las principales preocupaciones de las entidades que regulan su manejo para fines productivos sostenibles y de conservación, debido a la interacción de factores bióticos y abióticos que afectan los ecosistemas. Por decreto 698 del 18 de abril de 2000, el Ministerio de Medio Ambiente de la Republica de Colombia declaró, «El lago Guamuéz por su condición de humedal de importancia internacional, reconocido como riqueza ecológica de la humanidad, tiene aún más importancia para la zona de influencia, dadas sus características especiales al estar insertado en la cordillera de los Andes. Esto no implica que no pueda ser usado para algunas actividades antrópicas, como la pesca y la acuacultura, las que, por sus características, pueden llegar ser inocuas para el medio ambiente como alternativa adicional en la producción de alimento para la humanidad, pues en estas condiciones, el convenio Ramsar deja abierta la posibilidad de su uso para estos fines en condiciones racionales, siempre que no ofrezcan amenazas para su deterioro».

Por esta razón, los esfuerzos de investigación deben dirigirse a evaluar el impacto del hombre, sobre este importante cuerpo de agua y unir esfuerzos de variada índole que permitan conocer sus características limnológicas, bióticas, abióticas, vulnerabilidad y niveles de eutrofización, teniendo en cuenta que el lago se ha constituido en la única fuente de sustento económico para los moradores de la región, a través de turismo, pesca y acuacultura en estructuras flotantes y en un futuro próximo puede proveer agua potable a la ciudad de San Juan de Pasto, si se logra avanzar en el Proyecto Multipropósito que se propuso desde 1993 pero por falta de estudios ambientales adecuados y la ausencia de concertación con la comunidad no se ha podido desarrollar hasta la fecha. En consecuencia, la presente investigación pretende establecer mediante la metodología de muestreos periódicos cual ha sido el efecto de las explotaciones acuícolas en jaulas flotantes sobre las variables fisicoquímicas del lago Guamuéz, teniendo en cuenta que la cuenca ha sido intervenida totalmente por el hombre los bosques primarios son inexistentes hasta los 3.000 m.s.n.a y en las riberas del lago se encuentran varios asentamientos poblacionales de importancia como es el corregimiento del Encano que utiliza el río del mismo nombre, principal efluente del lago Guamuéz como vertedero de aguas servidas. Igualmente, las actividades antrópicas agrícolas, la erosión producto de la deforestación intensa con los consiguientes arrastres de materia orgánica, pesticidas y fertilizantes que llegan directamente e este cuerpo agua y que presumiblemente podrían causar serios problemas de eutrofización.

El actual proyecto puede ser un punto de partida para el desarrollo de una línea de investigación permanente, de tal manera que los datos obtenidos pueda generar información científica tendiente a desarrollar un plan de ordenamiento de la cuenca del lago Guamuéz con fines de conservación de la flora, fauna, explotación pecuaria, agrícola, recreativa y turística. Los estudios previos han demostrado que los niveles de contaminación del agua causados por la acuacultura son inferiores a las relacionadas con otras actividades agropecuarias por ser ésta una actividad que puede implementarse como producción limpia y amigable con el medio ambiente. Por las anteriores razones, la presente investigación pretende establecer el nivel de eutrofización a partir de las características limnológicas del Lago Guamuéz y la variación fisicoquímica espacial y temporal, registrada en diferentes sitos representativos, localizadas en las distintas riberas geográficas, donde se han implementado cultivos de trucha arcoiris en jaulas flotantes. Los resultados obtenidos permitirán a CORPONARIÑO y demás instancias institucionales y comunitarias relacionadas con el sector, establecer planes de administración y manejo sostenible y sustentable de la biodiversidad existente, con estrategias de gestión, autogestión y educación, que se dirijan al mejoramiento integral de la calidad de vida de los habitantes de la zona. 
La caracterización de los parámetros limnológicos del lago Guamuéz, permitirá establecer, el efecto de la producción de trucha arcoiris en jaulas flotantes, al grado de trofización de este ecosistema lenítico. El estudio se realizó, durante 90 días comprendidos de octubre a diciembre de 2008, período en el cual, se efectuaron 14 muestreos, en ocho estaciones principales (E) conformada cada una por tres sitios de lectura (S). La estimación paramétrica se implementó, mediante una sonda, electrométrica de profundidad, perfiles puntuales de temperatura $\left({ }^{\circ} \mathrm{C}\right)$, oxígeno disuelto $\left(\mathrm{mg} \cdot \mathrm{L}^{-1}\right), \mathrm{pH}$ $\left[\mathrm{H}^{+}\right]$, Demanda Bioquímica de Oxígeno (DBO5), nitratos. Así mismo, se registraron índice de turbidez y transparencia con Disco Secchi. Posteriormente, en los laboratorios especializados de la Universidad de Nariño y en el Laboratorio de Calidad de Aguas para Acuacultura del Programa de Ingeniería Acuícola, se determinaron según los protocolos establecidos por «Standard Methods for Examinations of Water and Wastes», la concentración de sólidos, nitritos, Fósforo total y amonio, por método volumétrico y se efectuaron los análisis de coliformes totales y fecales.

Por lo anteriormente expuesto, El presente estudio pretende evaluar, los parámetros limnológicos del lago Guamuéz, con el propósito de establecer, el efecto de la producción de trucha arcoiris en jaulas flotantes, en el grado de trofización de este cuerpo de agua.

\section{Metodología}

Caracterización limnológica del sistema lago Guamuéz en correlación con la producción de trucha arcoiris (Oncorhynchus mykiss), cultivada en jaulas flotantes.

\section{Localización}

El presente estudio se realizó en el Lago Guamuéz, corregimiento de El Encano, ubicado a 25 kilómetros al oriente la ciudad de San Juan de Pasto, en el departamento de Nariño, al suroccidente de la república de Colombia, con un volumen aproximado de 3.000 .000 .000 $\mathrm{m}^{3}$ de agua y coordenadas de $1^{\circ} 06^{\prime} 42,53^{\prime \prime}$ latitud Norte y $77^{\circ} 08^{\prime} 29.15^{\prime \prime}$ longitud Oeste, altura de $2.850 \mathrm{msnm}$ (metros sobre el nivel del mar) en la cota máxima y 2.745 msnm en la mínima, temperatura ambiental promedio de $11,6^{\circ} \mathrm{C}$ con variaciones entre 6 y $14^{\circ} \mathrm{C}$, humedad relativa del $86 \%$, brillo solar de 898,2 horas anuales y precipitación pluvial de $1.371 \mathrm{~mm}$ anuales. Figura 1.

Se desarrollaron durante la investigación 14 muestreos, en un período de 90 días comprendidos de octubre a diciembre de 2008, en ocho estaciones principales (E) conformada cada una por tres sitios de lectura denominadas así: SA (en el centro de cada explotación acuícola) SB (en la periferia) y SC (a $20 \mathrm{~m}$ del cultivo acuícola). Igualmente, se recolectaron las muestras para todos los

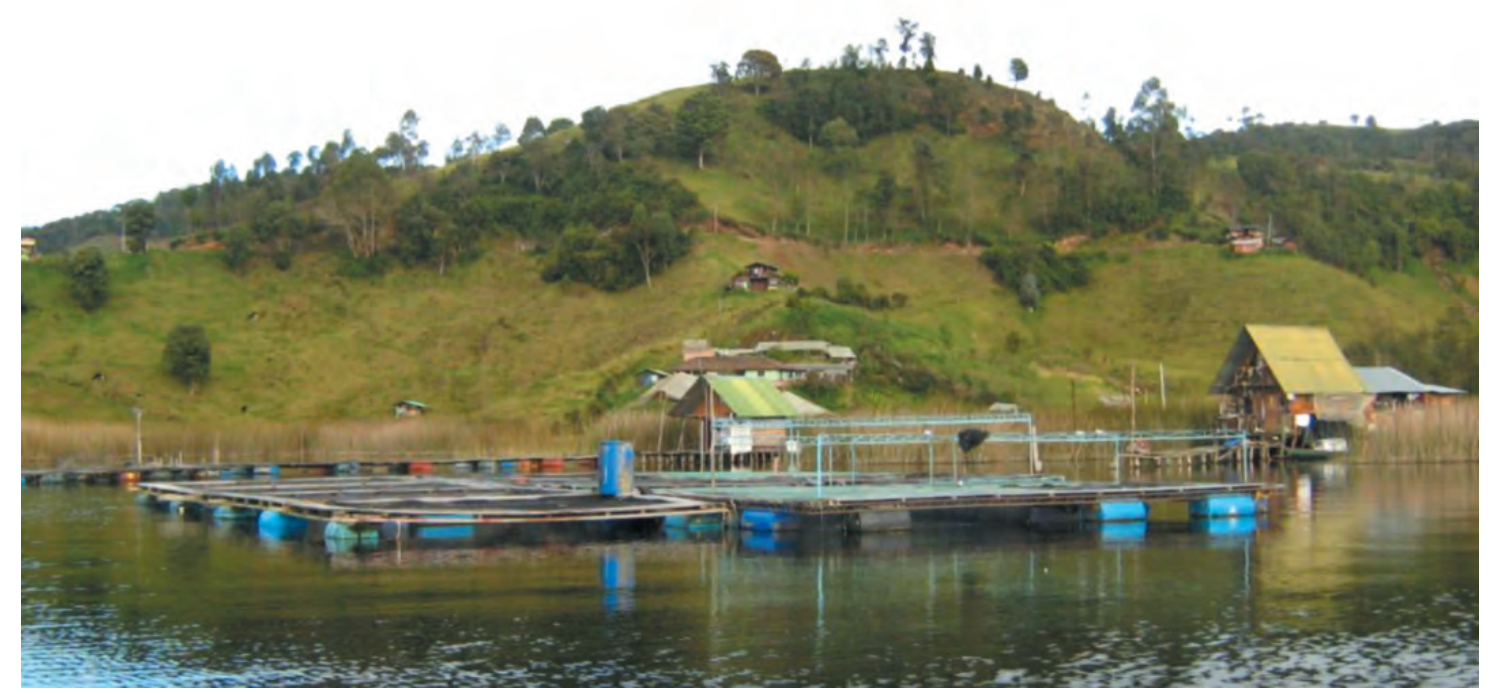

Fuente: Esta investigación.

Figura 1. Sistema de producción de trucha arcoíris en jaulas flotantes - Lago Guamuéz 
análisis de la superficie con excepción de bentos que debía hacerse del fondo del lago. Algunos exámenes como los de temperatura $\left({ }^{\circ} \mathrm{C}\right)$, oxígeno disuelto $\left(\mathrm{O}_{2} \mathrm{mg} \cdot \mathrm{L}^{-1}\right), \mathrm{pH}$ $\left[\mathrm{H}^{+}\right]$,y presión barométrica, requerían muestras adicionales de, profundidad media a 15 metros y otros como concentración de sólidos en suspensión, nitritos, ortofosfatos, amonio y bentos a la profundidad máxima permitida por la sonda de 30 metros. Las ocho estaciones principales, fueron referenciadas geográficamente con un equipo GPS (Garmin) y estaban conformadas por siete estaciones, distribuidas uniformemente en el perímetro de este cuerpo de agua y que se caracterizaban por poseer explotaciones acuícolas flotantes de diferente intensidad de producción y tecnología, contrastadas con una estación ubicada en el centro del lago sin presencia de jaulas flotantes con el fin de disponer durante la investigación de áreas representativas de diferente intensidad y efecto sobre las distintas variables limnológicas objeto del estudio, lo mismo que el impacto de vertimientos de aguas de origen antrópico y la existencia de afluentes y efluentes.

\section{Materiales, equipos y accesorios}

- Botella Muestreadora tipo Van Dom.

- Cámara de conteo de zooplancton tipo Sedgwick Rafter.

- EQUIPO YSI PROFESSIONAL PLUS compuesto por:

- Sonda Multiparametríca. Referencia 6050000.

- Sonda DBO. Referencia 5010.

- Sonda DO/pH/Temperatura. Referencia 6051020-30.

- Sonda Dual ISE/Temperatura. Referencia 6051010-30.

- Sensor DO Polarográfico. Referencia 605203.

- Sensor ISE de Amonio. Referencia 605104.

- Sensor ISE de Nitrato. Referencia 605106.

- Sensor ISE de Cloruro. Referencia 605105.

- Sensor de pH. Referencia 605101.

- Draga Ekman de 240,5 cm².

- Equipo GPS, Garmin.

- Balanza de precisión, Scout $600 \mathrm{~g}$ x 0,1 g-0722Sp601.

- Balanza de precisión, Scout 4000g x 0,1g - 2182Sp4001.
- Microscopio. NIKON OLIMPUS MODELO CX21F S1: 10XI, 40X y 100X.

- Estereoscopio. NICON SMZ1.

- Cámara de Microfotografía. SONY DSC - W80.7.2 MPX.

- Láminas porta y cubreobjetos.

- Cámara de Newbauer para conteo de fitoplancton.

- Camara Sedgewick-Rafter 2423M415

- Redes para plancton Con ojo de malla de 50 micras para Fitoplancton y de 200 micras Zooplancton.

- Frascos con capacidad de 500 y 700 mililitros.

- Pipetas de $1 \mathrm{~mL}$.

- Filtros, baldes graduados.

- Reactivos.

- Lugol al $4 \%$.

- Formol al 5\%.

- Aceite de inmersión.

- Materiales para etiquetado y registro.

- Cinta de enmascar.

- Marcador permanente.

- Registros.

- Etiquetas.

\section{Evaluación de parámetros fisicoquímicos}

La caracterización fisicoquímica de las aguas del lago, se realizó en la zona fótica de las ocho estaciones, durante los 14 muestreos, en los tres sitios de cada una de las estaciones (SA, SB y SC) con recolección de muestras para algunos análisis a nivel de superficie, profundidad media de la columna de agua a 15 metros y profundidad máxima permitida por la sonda de 30 metros.Para esto, se tomaron muestras con una botella recolectora tipo Van Dorn con capacidad de 2 litros. Un litro se destinó para el análisis fisicoquímico y el otro se utilizó para estudiar el plancton.) Para cada una de las ocho estaciones y en cada uno de los sitios de lectura, se implementaron perfiles puntuales de temperatura $\left({ }^{\circ} \mathrm{C}\right)$, oxígeno disuelto $\left(\mathrm{O}_{2} \mathrm{mg} \cdot \mathrm{L}^{-1}\right), \mathrm{pH}\left[\mathrm{H}^{+}\right]$, presión barométrica, Demanda Bioquímica de Oxígeno $\left(\mathrm{DBO}_{5}\right)$, nitratos utilizando una sonda, electrométrica, tipo YSI, y transparencia con Disco Secchi. Es importante aclarar que a partir de la lectura de disco Secchi, se reconvirtieron estos valores en Índice de Turbidez según Hela y Laveastu (1952), Posteriormente, en los laboratorios especializados de la Universidad de Nariño y en el Laboratorio de Calidad de Aguas para 
Acuacultura del Programa de Ingeniería Acuícola se determinaron concentración de sólidos en suspensión, nitritos, ortofosfatos y amonio, por método volumétrico Igualmente, se realizaron los análisis de Coliformes totales y fecales según los protocolos establecidos por «Standard Methods for Examinations of Water and Wastes». Figura 2.

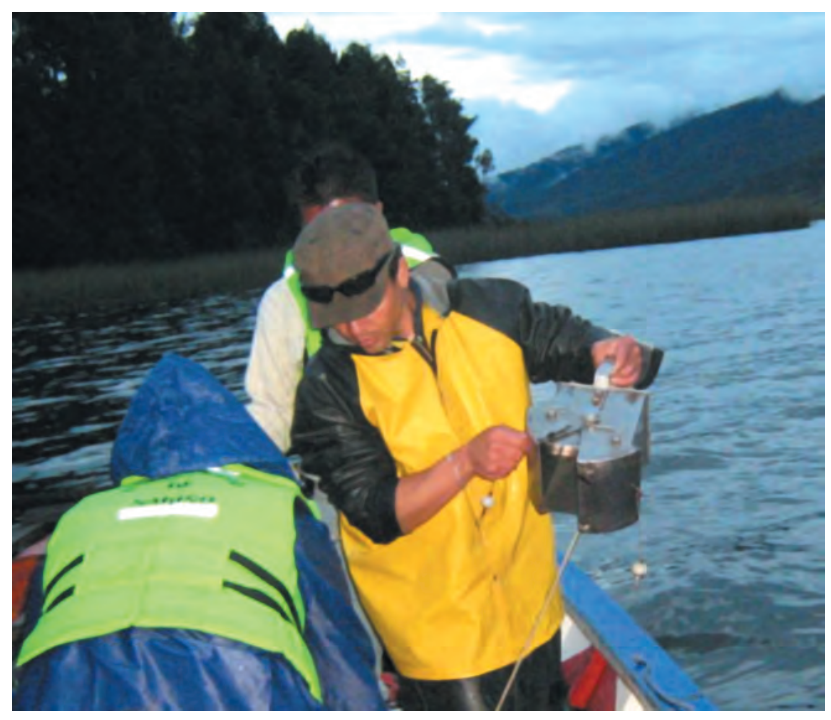

Fuente: Esta investigación.

Figura 2. Muestreo físico, químico y biológico en el Lago Guamuéz.

\section{Diseño experimental y análisis estadístico}

Se realizó un análisis de correlación de Pearson con las diferentes variables abióticas y bióticas, utilizando el paquetes estadístico Statgrapics versión 5.0 Windows.

\section{Resultados y discusión}

\section{Transparencia mediante disco secchi e índice de turbidez}

La estación que registra la mayor transparencia es la E5 que corresponde al centro del lago, la cual presentó un promedio de 4,65 metros e índice de turbidez de 0,37 según Hela y Laveastu (1952), seguido de la estación E1 con un promedio de 4,26 m en los diferentes sitios de lectura e índice de turbidez de 0,40\%, con un máximo de disco secchi de 4,34 m (0,39\% de turbidez) correspondiente al sitio C, (50 metros, calculados desde el borde de la estructura flotante, hacia el centro del lago), y un míni- mo de 4,21 m (0,42\% de turbidez) en SA (centro de las jaulas). (Tablas 1). En contraste, Los valores intermedios de área fótica e índice de turbidez, se establecen en las estaciones 2 y 6 con valores de 4,16 y 4,19 m e índice similar de turbidez de 0,41 y la estación que presenta la transparencia inferior y por ende el menor valor de turbidez es E3 con promedio de 3,85 m en los diferentes sitios de medida e índice promedio de turbidez de $44 \%$, con un máximo de transparencia de 3,96 m (0,43 m de turbidez) en el sitio SC y un mínimo de $3,79 \mathrm{~m}$ ( $0.45 \%$ de turbidez) en el punto SA. La menor transparencia, indica en un momento dado la distribución y estructura límite del ecosistema fótico lacustre que conllevan relaciones energéticas a partir de energía solar radiante como fuente de energía primordial del sistema, la cual se consume en el proceso de la fotosíntesis para sintetizar compuestos orgánicos carbonados. (Kormondy, 1994).

Los resultados del ensayo demuestran que la turbidez es superior en todas las estaciones, en el centro de las jaulas (SA) y disminuye a medida que las lecturas se alejan de las granjas flotantes. De tal manera que los mayores valores correspondientes a transparencia se encuentran sobre la isolínea que representa al punto $C$ en cada sitio de muestreo (Tabla 1). Igualmente, las explotaciones con mayor turbidez son las que disponen de número alto de jaulas y que se encuentran localizadas cerca de asentamientos urbanos como es el caso de la E8 localizada en la vereda El puerto. Este corregimiento carece de alcantarillado y sistema de tratamiento de aguas servidas y eliminación de excretas, las cuales se vierten directamente en el cauce del río El encano que es el mayor afluente de este cuerpo natural de agua, agravada esta situación debido a que la mayoría de moradores lavan directamente sus ropas en el río, utilizando detergentes ricos en fósforo, Además, existe la condición topográfica que las granjas flotantes de trucha localizadas cerca de la desembocadura del citado río se encuentran en sitios que no superan los 10 metros de profundidad. Lo que facilita los procesos de sedimentación y eutrofización litoral.

\section{Oxígeno disuelto}

En la E5 se detecta el valor máximo de 7,67 mg. $\mathrm{L}^{-1}$ y el valor mínimo de 6,38 en E8 y con base en los registros obtenidos del OD se detectan en superficie valores promedio en todas las estaciones y durante el período experimental de 7,6 mg. $\mathrm{L}^{-1}$ (Tabla 2) con un porcentaje de saturación de 71,24 lo cual se debe a los fenómenos 
Tabla 1. Transparencia y Turbidez de la Columna de agua, Hela y Laveastu (1952)

\begin{tabular}{lccc} 
Estación & Punto & Zona Fótica Promedio (m) & Turbidez (\%) \\
S1 & A & 4,21 & 0,40 \\
& B & 4,24 & 0,40 \\
S2 & C & 4,34 & 0,39 \\
& A & & 0,42 \\
& B & 4,07 & 0,42 \\
S3 & C & 4,01 & 0,39 \\
& & 4,42 & 0,45 \\
& A & & 0,45 \\
S4 & B & 3,81 & 0,43 \\
& C & 3,79 & 0,48 \\
& & 3,96 & 0,45 \\
S5 & A & & 0,41 \\
S6 & B & 3,57 & 0,37 \\
& C & 3,80 & 0,40 \\
& A & 4,17 & 0,42 \\
S7 & A & & 0,40 \\
& B & 4,65 & \\
S8 & C & & 0,44 \\
& & 4,26 & 0,42 \\
& A & 4,04 & 0,39 \\
& B & 4,28 & 0,56 \\
& C & & 0,53 \\
& A & 3,91 & 0,49 \\
\hline
\end{tabular}

Fuente: Esta investigación.

de mezcla del lago por oleaje, vientos y lluvias, lo que demuestra la naturaleza polimíctica del lago. Sin embargo, en la parte media de la columna se registra cantidades de $4,7 \mathrm{mg} \cdot \mathrm{L}^{-1}$ debido a la consistente sobresaturación en la superficie y a la utilización del mismo en la descomposición de la materia orgánica acumulada en el fondo. El oxígeno disuelto promedio del lago se presenta en condiciones aceptables, esto es, sobre los 7,26 mg..-1.

Es importante destacar que en algunos sitios de muestreo, la concentración de oxígeno disuelto baja gradualmente conforme aumenta la profundidad de la masa de agua. Esta condición se estima normal, debido a la reaireación superficial que se produce en todo el cuerpo de agua que se encuentra en contacto con la atmósfera, situación que es más evidente en los días con fuertes vientos, condición que se presentó repetidamente durante el estudio. Los resultados de oxígeno disuelto, están de acuerdo, con las mediciones realizadas en muchos lagos ecuatoriales de alta montaña, considerados oligotróficos donde se han encontrado concentraciones de oxígeno disuelto siempre mayores a $7 \mathrm{mg}^{-\mathrm{L}^{-1}}$. Como es el caso de los lagos Villa Rica en Chile; Alalay, Aricare y Blanca en Bolivia; Concepción, Cachimbo en Chile y Guachota y Hedional en Perú.

\section{Demanda bioquímica de oxígeno}

En la E2 se detecta un valor máximo de DBO5 de 7.20 mg.L-1 y mínimo de 6,69 en E8. En el centro del lago, el nivel medio es de 7,16 mg. $\mathrm{L}^{-1}$, en contraste, con el dato promedio de todos los sitios de muestreo y periodo de 
observación que fue de 7,08 mg. $\mathrm{L}^{-1}$ (Tabla 2). El mayor valor de $\mathrm{DBO} 5$ en $\mathrm{E} 8$, es debido a que esta granja flotante de trucha arcoiris, se encuentra cerca de la desembocadura del río el Encano que vierte gran cantidad de materia orgánica en el lago. Es importante destacar que el nivel promedio de oxígeno de este cuerpo de agua, es relativamente alto, superior a $7 \mathrm{mg} \cdot \mathrm{L}^{-1}$ lo que permite una descomposición gradual de la materia orgánica y debido a las dimensiones de este cuerpo de agua, actúa como una laguna de estabilización aerobia.

\section{Niveles de fósforo total}

Según Catalina y Blanca (2005). el fósforo es esencial para el crecimiento de los organismos y puede ser el elemento limitante de la productividad primaria de un cuerpo en el agua y estimula el crecimiento de micro y macroorganismos acuáticos fotosintéticos que en un momento dado puede causar afloramientos excesivos que causan sofocamiento de todos los organismos vivientes por bajas drásticas de oxígeno, produciendo la

Tabla 2. Parámetros Fisicoquímicos Promedios durante los muestreos en 8 Estaciones del Lago Guamuéz

\begin{tabular}{|c|c|c|c|c|c|c|c|c|c|}
\hline Parámetro & Ubicación & E1 & E2 & E3 & E4 & E5 & E6 & E7 & E8 \\
\hline Oxígeno disuelto (mg/L) & Superficie & 7,31 & 7,22 & 7,10 & 7,50 & 7,67 & 7,52 & 7,47 & 6,31 \\
\hline Temperatura $\left({ }^{\circ} \mathrm{C}\right)$ & Superficie & 16,53 & 16,57 & 16,48 & 16,60 & 16,62 & 16,67 & 16,46 & 16,11 \\
\hline $\mathrm{pH}$ & Superficie & 6,55 & 6,33 & 6,77 & 6,87 & 6,67 & 5,96 & 6,24 & 6,45 \\
\hline \multirow[t]{2}{*}{ Fósforo (mg P-PO4/L) } & Superficie & 0,16 & 0,00 & 0,00 & 0,00 & 0,00 & 0,00 & 0,00 & 0,00 \\
\hline & Fondo & 0,00 & 0,00 & 0,00 & 0,00 & 0,00 & 0,00 & 0,00 & 0,05 \\
\hline \multirow[t]{2}{*}{ Nitritos (mg N - NO2/L) } & Superficie & 0,00 & 0,00 & 0,00 & 0,00 & 0,00 & 0,00 & 0,00 & 0,00 \\
\hline & Fondo & 0,00 & 0,00 & 0,00 & 0,00 & 0,00 & 0,00 & 0,00 & 0,00 \\
\hline \multirow[t]{2}{*}{ Nitratos (mg N - NO3/L) } & Superficie & 0,36 & 0,37 & 0,57 & 0,30 & 0,47 & 0,23 & 0,31 & 0,54 \\
\hline & Fondo & 0,50 & 0,53 & 0,57 & 0,52 & 0,37 & 0,45 & 0,59 & 0,50 \\
\hline Sólidos Totales (mg/L) & Superficie & 51,43 & 52,51 & 44,10 & 47,22 & 42,70 & 42,07 & 50,84 & 55,03 \\
\hline Sólidos Totales (mg/L) & Fondo & 49,94 & 47,42 & 293,40 & 78,66 & 45,50 & 142,01 & 58,01 & 121,79 \\
\hline Sólidos disueltos (mg/L) & Superficie & 34,23 & 34,67 & 35,77 & 33,33 & 35,10 & 35,44 & 38,72 & 38,44 \\
\hline Sólidos disueltos (mg/L) & Fondo & 38,87 & 33,44 & 33,14 & 44,10 & 35,15 & 37,46 & 38,83 & 59,16 \\
\hline Sólidos suspendidos (mg/L) & Superficie & 21,71 & 23,02 & 13,60 & 17,66 & 10,75 & 10,34 & 16,42 & 22,89 \\
\hline Sólidos suspendidos (mg/L) & Fondo & 15,47 & 18,67 & 286,18 & 39,06 & 14,37 & 117,38 & 24,44 & 73,57 \\
\hline Sólidos Volátiles (mg/L) & Superficie & 14,10 & 10,30 & 10,00 & 13,90 & 8,50 & 5,60 & 11,30 & 11,70 \\
\hline Sólidos Volátiles (mg/L) & Fondo & 11,60 & 17,10 & 283,90 & 17,20 & 6,60 & 11,40 & 14,20 & 20,50 \\
\hline \multirow[t]{2}{*}{ Amonio } & Superficie & 0,06 & 0,03 & 0,04 & 0,04 & 0,02 & 0,04 & 0,03 & 0,05 \\
\hline & Fondo & 0,06 & 0,04 & 0,03 & 0,03 & 0,02 & 0,02 & 0,01 & 0,01 \\
\hline \multirow[t]{2}{*}{ Dureza } & Superficie & 1,25 & 1,45 & 1,55 & 1,20 & 0,02 & 0,02 & 0,01 & 0,01 \\
\hline & Fondo & 0,06 & 0,04 & 0,03 & 0,03 & 0,02 & 0,02 & 0,01 & 0,01 \\
\hline \multirow[t]{2}{*}{ Alcalinidad } & Superficie & 0,06 & 0,03 & 0,04 & 0,04 & 0,02 & 0,04 & 0,03 & 0,05 \\
\hline & Fondo & 0,06 & 0,04 & 0,03 & 0,03 & 0,02 & 0,02 & 0,01 & 0,01 \\
\hline \multirow[t]{2}{*}{ OD \% } & Superficie & 71,17 & 71,70 & 71,17 & 71,43 & 72,07 & 73,11 & 71,51 & 67,79 \\
\hline & Fondo & 71,89 & 72,31 & 71,09 & 71,79 & 74,51 & 73,57 & 72,13 & 63,83 \\
\hline \multirow[t]{2}{*}{ DBO mg/L } & Superficie & 7,10 & 7,20 & 7,18 & 7,16 & 7,13 & 7,15 & 7,06 & 6,69 \\
\hline & Fondo & 7,21 & 7,36 & 7,07 & 7,12 & 7,38 & 7,18 & 7,18 & 6,34 \\
\hline
\end{tabular}

Fuente: Esta investigación. 
muerte de los lagos p procesos de eutrofización intensa como es el caso de los grandes lagos africanos y en Suramérica el lago Nabor en Argentina. También se ha observado que en aguas de alta alcalinidad con exceso de sodio y pobre de calcio, generalmente de poca profundidad queda una concentración insuficiente de calcio para la coprecipitación del fosfato cálcico con el carbonato de calcio que es uno de los mecanismos de autopurificación de fosfato más eficientes en cuerpos de agua. En contraste, en aguas con baja a media reserva alcalina; el ortofosfato permanece en solución en altas cantidades (Margalef, 1983),

Con respecto a Fósforo Total, las mediciones realizadas en las diferentes estaciones y sitios de muestreo indican valores no detectables por los protocolos y equipos de laboratorio utilizados en este estudio y solo se cuantificó en la estación E1 con un valor medio de 0,16 mg. $\mathrm{L}^{-1}$ (Tabla 2). Es importante aclarar que la granja flotante, existente en esta zona, recibe el arrastre de material, procedente de una microcuenca deforestada, caracterizada por cultivos limpios de papa y cebolla.

\section{Niveles de nitrógeno total}

Con relación a los niveles de nitrógeno Kjeldahl, amoniacal, orgánico, en la E1 se detecta el valor máximo de $0,06 \mathrm{mg} \cdot \mathrm{L}^{-1}$, el mínimo de 0.02 en el centro del lago (E5) y un dato medio de 0,04 durante el periodo de investigación. Los niveles de amonio son relativamente bajos para un cuerpo de agua de las condiciones fisiográficas señaladas. Sin embargo, en un posible proceso de eutrofización futuro, los niveles podrían subir a valores subletales o letales para los organismos acuáticos, causando impactos negativos sobre la cadena trófica de este ecosistema lacustre.

La cuantificación de nitritos no fue detectable en las zonas de muestreo debido a los procesos de oxidoreducción que sufre este elemento Con respecto a nitratos en la E3 se detecta el valor máximo de $0,57 \mathrm{mg} \cdot \mathrm{L}^{-}$ ${ }^{1}$ y el valor mínimo de 0,23 en E6 y un valor promedio general durante todo el periodo de observación de 0,39. En contraste con el centro del lago que registra un dato

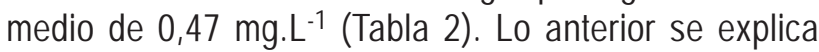
debido a que la E3, se encuentra localizada en la vereda Santa Lucia, lugar de vaciamiento del lago a través del río Guamuéz. Los datos anteriores concuerdan con evaluaciones realizadas en lagos y lagunas del Perú que reportan niveles de nitrógeno total estuvo sobre los $0,20 \mathrm{mg} \cdot \mathrm{L}^{-1}$.

\section{Relación nitrógeno total: fósforo total}

La relación Nitrógeno Total: Fósforo Total, según los datos promedios de estos nutrientes registrados en todas las estaciones y sitios de muestreo durante el periodo de observación fue de 2,6:1 (Tabla 2). Lo anterior, demuestra que el elemento nutricional limitante en la futura eutrofia del lago es Fósforo. El fósforo y nitrógeno, se encuentran en el lago, predominante en forma de fosfatos y nitratos y este último es mayor en los sitios de muestreo que están cerca de la desembocadura de afluentes que aportan aguas servidas como es el caso del río El encano y las quebradas Moras, Romerillo y Quilinza, a las cuales les llegan escorrentías que arrastran gran cantidad de materia orgánica, nutrientes y fertilizantes, procedentes de cultivos limpios de mora, papa y gramíneas para explotación de ganado y cuyes (Cavia porcellus). También ha contribuido al aporte de nitrógeno, la construcción de la carretera perimetral entre las veredas Motilón y Ramos que aportan materiales en suspensión incrementando la turbidez y disminuyendo la transparencia, específicamente durante el periodo de lluvias. En contraste, la contaminación generada por las explotaciones de truchas en estructuras flotantes, existentes en el lago Guamuéz, está representada por las excretas de las truchas, la evisceración de los ejemplares cosechados, directamente sobre las aguas del lago y el alimento no consumido por los peces que se depositan directamente en el fondo del cuerpo de agua, sufriendo procesos de descomposición aerobios y anaerobios que liberan gradualmente en la columna de agua, gran cantidad de fósforo y nitrógeno debido a los niveles de harina de pescado y torta de soya que poseen las dietas artificiales para organismos hidrobiológicos de cultivo (López et al., 2005).

\section{Sólidos}

En esta investigación, se calcularon sólidos totales, sólidos disueltos y sólidos en suspensión Con respecto a sólidos totales en la E8 se detecta el valor máximo de 55,03 $\mathrm{mg} . \mathrm{L}^{-1}$, lo cual se explica porque esta granja flotante se encuentra cerca de la desembocadura del río Guamuéz y el mínimo de $42,07 \mathrm{mg}^{-L^{-1}}$ en E6 que se encuentra localizada en la vereda Santa Teresita, donde se presentan las mayores profundidades promedias de la columna de agua, ideales para el cultivo de peces. En este sitio el lago actúa como cuerpo de sedimentación con un dato medio de 48,24 $\mathrm{mg} . \mathrm{L}^{-1}$ en todas las estaciones sitios de muestreo durante el periodo de análisis, en contraste con el centro del lago que registra un valor de 42,7 mg.L-1 (Tabla 2). 
Con relación a sólidos disueltos en la E7 se detecta el valor máximo de 38,72 mg.L-1 el mínimo de $33,33 \mathrm{mg.L} \mathrm{L}^{-1}$

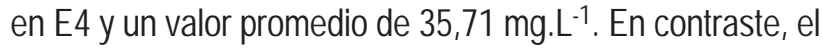
centro del lago registra un dato medio de 35,1 mg..-1. Con relación a sólidos suspendidos en la E2 se establece el valor máximo de 23,02 mg. $\mathrm{L}^{-1}$ y mínimo de $10.34 \mathrm{mg} . \mathrm{L}^{-1} \mathrm{en}$ E6 $y$ el promedio de $35,71 \mathrm{mg} \cdot \mathrm{L}^{-1}$ en todas las estaciones $y$ sitios de muestreo. En contraste, el centro del lago registra un dato medio de 10,75 mg..-1 (Tabla 2). Con respecto a sólidos volátiles, en la E1 se registró el valor máximo de 14,1 mg.L-1-1 y mínimo de 5,6 mg..-1 en E6 y un dato medio de 19,68 mg. $\mathrm{L}^{-1}$. En contraste, el centro del lago presenta un valor promedio de $8,5 \mathrm{mg}^{-\mathrm{L}^{-1}}$ (Tabla 2).

Según los datos arriba cuantificados, la presencia de sólidos totales flotantes y suspendidos no es importante a lo largo de todo el lago, aunque en la estación ocho, se presentan un gran número de sólidos suspendidos que disminuyen la visibilidad del disco Sechii, lo cual no permite en gran parte la entrada de la luz solar, disminuye la fotosíntesis debido a la descarga de sólidos que lleva el río El Encano.

\section{Alcalinidad}

La alcalinidad total, mide directamente la reserva alcalina, es decir, la suma de aniones procedentes directa 0 indirectamente a través de las disoluciones de las sales correspondientes, de tal manera que en la E1 se detecta un valor máximo de $18 \mathrm{mg} \cdot \mathrm{L}^{-1}$ y mínimo de 16 $\mathrm{mg. \textrm {L } ^ { - 1 }}$ en E7 con un dato medio de $16,75 \mathrm{mg}^{-\mathrm{L}^{-1}}$. Tales cifras señalan una reserva alcalina intermedia, cuyo incremento estaría determinado por las características del suelo del lago, proceso intensificado probablemente por la mezcla de la masa de agua y el oleaje al acarrear partículas hacia el interior del lago, lo que produce un cambio de bicarbonatos a carbonatos que de acuerdo con Wetzel (1975) a niveles mayores de 9,5 el carbonato comienza a adquirir preponderancia en la alcalinidad del cuerpo de agua. Además a un incremento del pH, el $\mathrm{Ca}^{++}$decrece y los cationes principales en solución de $\mathrm{Mg}^{++}, \mathrm{Na}^{++}$y en ocasiones, cantidades apreciables de $\mathrm{K}^{+}$, pueden afectar la alcalinidad del agua (Tabla 2).

\section{Dureza}

Es el total de sales solubles en forma de carbonato y/o bicarbonatos existentes en un cuerpo de agua. La investigación demostró que la estación E7 registra el valor máximo de 21 ppm y el mínimo de 14 ppm en E4 con un valor promedio de $17,19 \mathrm{mg} \cdot \mathrm{L}^{-1}$ durante el periodo de observación (Tabla 2).

\section{Temperatura}

Con relación a Temperatura en la E6 se detecta un valor máximo de $16,6^{\circ} \mathrm{C}$, un mínimo de $16,1^{\circ} \mathrm{C}$ en la estación E8 con un dato promedio de $16,5^{\circ} \mathrm{C}$, durante todo el periodo de observación (Tabla 2).

\section{Potencial de Hidrógeno (pH)}

Con respecto a $\mathrm{pH}$ la $\mathrm{E} 4$ registra un valor máximo de 6,87 y mínimo de 5,96 en la E6 con un dato medio de 6,48 (Tabla 2).

\section{Caracterización Fisicoquímica Comparativa del Lago Guamuéz en los Años 1996-2008}

La caracterización fisicoquímica del lago Guamuéz, realizada por el Grupo de Investigaciones Acuícola (GIAC) en el 2008, al compararla con los datos analizados por el estudio de Castillo y Martínez (1996), en varias granjas flotantes de trucha arcoiris, localizadas en este cuerpo de agua (Tabla 3), se encuentra que en la estación Romerillo (E1), el $50 \%$ de los parámetros evaluados son mayores en 1996 y en el caso concreto del pH, se incrementó en 0,31 unidades; la $\mathrm{DBO}_{5}$ representa en 2008 el 61,74\% del registro de 1996 (11,50 vs 7,10 mg. L-1); fósforo total en 2008 simboliza el $80,67 \%$ del valor encontrado en 1996 (0,20 vs $0,16 \mathrm{mg} \cdot \mathrm{L}^{-1}$ ) y los niveles de nitratos son para 2008 el 3,43\% los miligramos cuantificados en 1996 (10,50 vs $0,36 \mathrm{mg} . \mathrm{L}^{-}$ 1). Las variables de la investigación realizada en el 2008, son superiores con relación a temperatura que registró un incremento de $22,44 \%$ ( 13,50 vs. $\left.16,53^{\circ} \mathrm{C}\right)$, los niveles de oxígeno disuelto se elevaron en $7,50 \%$, pasando de 6,80 a 7, $31 \mathrm{mg} . \mathrm{L}^{-1}$. Con relación a la estación Santa Lucia (E3), los parámetros en 2008 se incrementaron en 70\%; es destacable referenciar el incremento de la temperatura y la transparencia del cuerpo de agua en $11,10 \%$ para los dos variables. Igualmente, el oxígeno disuelto incremento en $4,18 \%\left(6,82\right.$ vs $\left.7,10 \mathrm{mg}^{-L^{-1}}\right)$. Los niveles de $\mathrm{DBO}_{5}$ en 2008 representan el $94,16 \%$ del valor registrado en 1996 (7,63 vs 7,18 mg.L-1). Los nitratos en 1996 están en $94 \%$ por encima de los niveles de 2008 (9,47 vs 0,57 mg. L-1 $)$. Tabla 3.

La estación de muestreo ubicada en el centro del lago Guamuéz, (E5) registra mayores valores para las variables temperatura, $\mathrm{OD}, \mathrm{DBO}_{5}$ y amonio de tal manera que 
Tabla 3. Análisis Comparativo Investigaciones Limnológicas 1996 y 2008

\begin{tabular}{lcccccc|}
\hline Zona de muestreo & \multicolumn{2}{c}{ Romerillo } & \multicolumn{2}{c}{ Santa Lucía } & \multicolumn{2}{c}{ Centro / Lago } \\
\hline Parámetros & 1996 & 2008 & 1996 & 2008 & 1996 & 2008 \\
pH & 6,86 & 6,55 & 6,76 & 6,77 & 6,77 & 6,67 \\
Transparencia & 4,72 & 4,26 & 14,83 & 16,48 & 5,20 & 4,65 \\
Temperatura & 13,50 & 16,53 & 14,83 & 16,48 & 13,33 & 16,62 \\
O $_{2}$ Disuelto & 6,80 & 7,31 & 6,82 & 7,10 & 7,39 & 7,67 \\
DBO 5 & 11,50 & 7,10 & 7,63 & 7,18 & 10,25 & 7,38 \\
DBO/DQO (\%) & 27,83 & 71,17 & 14,86 & 71,17 & 27,56 & 72,07 \\
Fosfatos & 0,20 & 0,16 & 0,18 & $<L D$ & 0,07 & $<$ LD \\
Nitritos & 0,26 & $<$ LD & 0,16 & $<$ LD & 0,33 & $<$ LD \\
Nitratos & 10,50 & 0,36 & 9,47 & 0,57 & 4,40 & 0,47 \\
& & & & & &
\end{tabular}

Fuente: Esta investigación.

la temperatura se elevó de $13,33^{\circ} \mathrm{C}$ a $16,62^{\circ} \mathrm{C}(24,65 \%)$ y Oxígeno Disuelto de 7,39 mg..-1 a 7,67 mg..-1 en 2008. En 1996, son superiores los valores de $\mathrm{pH}$, transparencia, $\mathrm{DBO}_{5}$ y Nitratos con relación a 2008, en niveles de 98,47, $89,42,72$ y 10,68 respectivamente. Sin embargo se deben explicar estas diferencias debido a que las investigaciones fueron realizadas en periodos climáticos diferentes.

\section{Conclusiones y recomendaciones}

\section{Conclusiones}

- Los análisis demuestran que la transparencia es menor en todas las estaciones, en el centro de las jaulas (SA) y disminuye a medida que las lecturas se alejan de las granjas flotantes (SC). Igualmente, las explotaciones con mayor turbidez, son las que disponen de número alto de jaulas y que se encuentran localizadas cerca de asentamientos urbanos como es el caso de la E8 en la vereda El puerto.

- En la E5, se detecta el valor máximo de OD de 7,67 mg.L-1 y el valor mínimo de 6,38 mg.L-1 en E8 que concuerda con valores superiores de $\mathrm{DBO}_{5}$ de 7,20 mg.L ${ }^{-1}$ en E8 y mínimos de 6,69 en E2.
- El mayor número de coliformes totales y fecales se presenta en las estaciones próximas a la desembocadura del río el Encano, es decir las estaciones E7 y E8.

- La máxima temperatura promedia se registra en la E6 con un valor de $16,6^{\circ} \mathrm{C}$, y mínimo de $16,1^{\circ} \mathrm{C}$ en la estación E8, debido al mayor nivel de turbidez.

- La relación Nitrógeno Total: Fósforo Total, según los datos promedios de estos nutrientes, en todas las estaciones y sitios de muestreo, durante el periodo de observación fue de 2.6:1 Lo anterior, demuestra que el elemento nutricional limitante para una futura eutrofia del lago es el Fósforo.

- El nivel de nitrógeno es mayor en los sitios de muestreo que están cerca de la desembocadura de afluentes que tiene gran parte de sus cauces, en zonas deforestadas o de potreros como el río El encano y las quebradas Moras, Romerillo y Quilinza, a las cuales les llegan escorrentías que arrastran gran cantidad de materia orgánica, nutrientes y fertilizantes, procedentes de cultivos limpios de mora, papa y explotaciones pecuarias.

- Los niveles de nitrógeno, también son relativamente superiores, en las estaciones donde se 
encuentra las jaulas flotantes de mayor intensidad de producción, debido a las excretas de las truchas, los flujos procedentes de las salas de evisceración que en algunas explotaciones, llegan directamente al lago y el alimento, no consumido por los peces que se acumulan en el fondo del cuerpo de agua, sufriendo procesos de descomposición aerobios y anaerobios que liberan gradualmente en la columna de agua, fósforo y nitrógeno.

- El Índice de Estado Trófico de Carlson (IET) es de 32,89 , que demuestra la oligotrofía de este lago, el cual coincide con los valores fisicoquímicos, niveles de Fósforo y de productividad primaria registrados en los distintos sitios de muestreo.

- El análisis de Pearson demuestra que existen correlaciones altamente significativas entre la abundancia a nivel de superficie de cianofitas, rotíferos y copépodos con fósforo total $(0,74)$. Igualmente, correlación positiva entre protozoarios y clorofila - a $(0,81)$. Las categorías biológicas del bentos presentan correlaciones superiores en las variables de diatomeas con sólidos disueltos de fondo $(0,64)$. Cianofitas con nitratos $(0.76)$, sólidos totales, sólidos suspendidos, sólidos volátiles medidos en profundidad de 10 metros con índices de $0,78,0,78$ y 0,76 respectivamente.

- Los valores fisicoquímicos y bacteriológicos que registra el lago Guamuéz, son adecuados para el desarrollo de actividades agrícolas y pecuarias, según la reglamentación vigente.

- Al evaluar el comportamiento del lago durante un período de 12 años comprendidos entre 1996 y 2008, teniendo en cuenta los resultados de esta investigación, comparados con el estudio de Castillo y Martínez (1996), en varias granjas flotantes de trucha arcoiris, localizadas en este cuerpo de agua, se evidencia que no se ha presentado un deterioro negativo, en la caracterización fisicoquímica y por ende de la productividad primaria, causado por las actividades acuícolas, considerando la capacidad de autopurificación de este cuerpo de agua por su condición polimíctica temporal Sin embargo, esta capacidad podría estar limitada en el futuro, debido a las condiciones oligotróficas del mismo.

\section{Recomendaciones}

- Implementar de manera urgente un plan de ordenamiento integral del lago Guamuéz que incluya todas las actividades pesqueras, acuícolas, forestales, agrícolas, pecuarias y la disposición sanitaria de aguas servidas, procedentes de los distintos asentamientos urbanos, localizados en la zona de influencia para disminuir el aporte de materia orgánica en este importante lago de altiplano.

- Replicar el estudio realizado en un periodo climático diferente con el fin de contrastar la información de invierno a verano.

- Ordenar el traslado de las explotaciones de trucha arcoiris que se encuentran en profundidades promedias inferiores a $5 \mathrm{~m}$ con el fin de evitar la liberación de lodos y nutrientes que afectarían este sistema lacustre.

- Promover con las casas fabricantes de alimentos artificiales para peces, la utilización de fuentes de fósforo de alta biodisponibilidad con el fin de permitir un menor nivel en las dietas.

- Implementar las mallas de colecta de alimento sobrante en cada una de las granjas flotantes con el fin de disminuir el efecto del Fósforo y el Nitrógeno de las dietas artificiales y su interacción con el agua y el fondo del lago Guamuéz.

- Establecer una moratoria en los permisos de concesión de nuevos proyectos acuícolas flotantes hasta que se implemente el sistema de disposición de aguas servidas en los asentamientos urbanos localizados en el lago Guamuéz.

\section{Referencias bibliográficas}

Buchelli, L. Limnología del lago Guamuéz y sus posibilidades para la acuacultura. Pasto. Colombia: Universidad de Nariño. 16 p.

Casallas J. y Gunkel G. 2001. APHA. 1989. Standard Methods for the Examination of Water and Wastewater. American Public Health Association, Washington, D.C. 17th Ed. 1550 pp, 1992. 
Castro, R. y Montero, R. Análisis hidrológico básico de la cuenca del lago Guamuéz como alternativa de fuente de abastecimiento al déficit de agua en Pasto. Pasto, Colombia: Universidad de Nariño, Facultad de Ingeniería Civil, 1989.

Conde-Porcuna, E. Ramos-Rodríguez, R. MoralesBaquero. El zooplancton como integrante de la estructura trófica de los ecosistemas lénticos. Ecosistemas revista científica y técnica de Ecología y medio Ambiente http:/l www.revistaecosistemas.net/pdfs/8.pdf

Corporación Autónoma Regional de Nariño (CORPONARIÑO), Plan de ordenamiento y manejo integral de los recursos naturales de la cuenca alta del río Guamuéz. Pasto, Colombia, 1994.

Corporación Autónoma Regional de Nariño (CORPONARIÑO). Diagnóstico acuícola de la cuenca alta del río Guamuéz. Pasto, Colombia, 2004.

Ducharme, A. Estudio Fisicoquímico y biológico del lago de Tota. Revisión bibliográfica de la Empresa de Acueducto y Alcantarillado de Bogotá, 1975.

Donato. J. C. Fitoplancton y aspectos físicos y químicos de la laguna de Chingaza (Una laguna tropical de alta montaña). Cuad. Divulg., 11(1991):1-18.

García, R.; Mora, A. y Rodríguez, H. Evaluación de parámetros físico - químicos como indicadores del grado de eutrofización del lago Guamuéz, municipio de Pasto, Nariño, Colombia. Pasto, Colombia: Universidad de Nariño, Programa de Ingeniería en Producción Acuícola. 108 p., 2001.

Gunkel, G. y J. Casallas. Limnnology oj an Ecuatorial High Mountain Lake, Lago San Pablo, Ecuador: Limitation of Lake Productivity by Deep Diurnal Mixing. Proceedings of the internacional Association of Theoretical and Applied Limnology. (en prensa), 2001.

Holdrige, L. Ecología basada en las zonas de vida. Instituto Interamericano de Ciencias Agrícolas. San Jose, Costa Rica, 1978.

Hutchinson, G. E. Y H. Lofler. The termal classification of lakes. Pro. Nut. Acad. Sci., 42, 1956.

Hupfer, M. 1995. Bindungsformen und Mobilität des Phosphors in Gewässersedimenten. In C. Steinberg, H. Bern- hard \& H. Klapper (eds.). Handbuch «Angewandte Limnologie». Ecomed, Landsberg, IV-3-2:(1956)1-22. 767 p.

Instituto de Hidrología Meteorología y Estudios Ambientales (IDEAM). Registros meteorológicos: Estación climatológica principal El Encano. Pasto, Colombia. 12 p., 2000.

Kormondy, E. Conceptos de Ecología Aplicada. . Madrid, España: Alianza Editorial, 1994.

Loffler, $\mathrm{H}$. The limnology of tropical highmountain lakes. Verh. Interant. Verein. Limnol., 15: Ecol. S'St., 18(1964): 159-184. (2/3):145-207. 176-193.

Loffler, H. Zur Ostrakoden- und Copepodenfauna Ekuadors. Arch. Hydrobiol., 59(1963):196-234.

López, J. Nutrición Acuícola. Editorial Universitaria. Universidad de Nariño. Pasto. 211 p. 1997.

López, J., Mena P., Torres C., Rodríguez J., Imuez M. Y Burgos A., Evaluación de inmunoestimulantes en las fases de levante y ceba de trucha arcoiris (O. mykiss) cultivada en jaulas flotantes en el Lago Guamuéz. Vicerrectoria de Investigaciones Postgrados y Relaciones Internacionales. Pasto. Colombia: Sistema Investigaciones. p. 6, 2005.

Lewis, W. M. Jr. The thermal regime of lake Lanao (Philippines) and its theoretical implications for tropical lakes. Limnol. Oceanogr., 18(2)(1973):200-217.

Margalef R. Limnología. Ed. Omega Barcelona Espaก̃a. (1983):779-867.

Marshall D. Biología de las Algas, Enfoque fisiológico. Departamento de Botánica. Universidad de Georgia: Noriega Editores. Primesa Edición. (1987):11-25.

Melack, J. M. Recent developments in tropical limnology. Verh. Internat. Verein. Limnol., 26, 1996.

Muñoz P. y Obando M. Clasificación taxonómica densidad y diversidad de plancton existente en el lago Guamuéz, corregimiento del Encano.Municipio de Pasto, Nariño, Colombia. 16 p, 2000.

Narváez, A. Estudio batimétrico y algunas relaciones ecológicas del lago Guamuéz. Universidad de Nariño, Departamento de Biología. Pasto, 1984. 41 p. 
Nilssen, J. P. Tropical Lakes - Functional ecology and hture development: The need for a process oriented approach. Hydrobiologia, 11(3)(23)(1984):1-242.

Payne, A. I. The ecologv of tropical lakes and rivers. John Wiley \& Sons, Manchester. 1986. 301 pp.

Pérez, J. y N, Maclean. The haemoglobins of the fish Saratherodon mossambicus. Functional significance and ontogenic changes. J. Fish. Biol. 9(1976):447-485.

Powell, T., M. H. Kirkish, P. J. Neale, \& RichersoN, P., J. The diurnal cycle of stratification in Lake Titicaca: Eddy diffusion. Verh. Internat. Verein. Limnol. 22(1984):12371243.

Ramírez, A, G. Ecología Métodos de Muestreo y Análisis de Poblaciones y Comunidades. Editorial Pontificia Universidad Javeriana. 2006.

Ramírez, J. y Machado. Influencia de la precipitación y la ortofosfatos sobre el fitoplancton de la represa de la Fe. Act. Biol. 11(39)(1982):3-21.

Richerson, P. J., P. J. Neale et al. Patterns of temporal variation in lake Titicaca. A high altitude tropical lake. I. Background physical and chemical processes and primary production. Hidrobiologia, 138(1986):205-220.

Richerson, P. J. Lake Titicaca. A Síntesis of Limnological Knowledge. Kluwer Academia Publishers, 1992.

Roldán Pérez G. Guía para el estudio de los macroinvertebrados Acuáticos del Departamento de Antioquia. Fondo Fen Colciencias. Universidad de Antioquia. Bogotá Colombia 1988. 1-6 p.
Roldán, G. Fundamentos de Limnología Neotropical. Editorial Universidad de Antioquia, Medellín. 1992. $529 \mathrm{pp}$.

Roldán P, G. A. Estudio limnológico del embalse de el Guavio Colombia. IRevista de la Academia Colombiana de Ciencias Exactas, Físicas y Naturales (Santafe de Bogotá) Mar. 24(090)2000:73-84.

Roldán P, G. A. y Posada G, J. A. Estudio limnológico de los recursos hídricos del Parque de Piedras Blancas. IBogotá: Academia Colombiana de Ciencias Exactas, Físicas y Naturales. 2001.

Sipauba-tavares, L. H. y Rocha, O. Producáo de plancton (Fitoplancton e zoopláncton) para alimeníacao de organismos acuáticos. Sao Carlos: Rima, Fapesp. 2003. $106 \mathrm{p}$.

Steinitz-Kannan, M., Colinvaux, P. A. y Kannan, R. Limnological Studies in Ecuador 1. ASurvey of chemical and physical properties of Ecuadorian lakes. Archiv Hydrobiol. Supplement 65(1983):61-105.

Vollenweider, R. A. Scientific Fundamentals of the Eutrophication of Lakes and Flowing Waters, with Particular Reference to Nitrogen and Phosphorus as Factors in Eutrophication. OECD Reporte DASiCSli68.27. 1968. 192 pp.

Wetzel, R. G. Limnology. Saunders College, Philadelphia. 1983. $767 \mathrm{p}$.

Wetzel R. G. Limnology. W. B. Saunders Co. Philadelphia USA. (1975):71-79. 